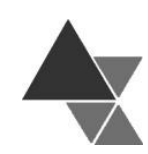

\title{
Caracterização do consumo alimentar e estado nutricional de feirantes do município de Dourados - Mato Grosso do Sul, Brasil
}

\author{
Valéria dos Reis Narciso1, Caroline Momente Martins Saturnino², Naiara Ferraz \\ Moreira $^{3}$ e Verônica Gronau Luz ${ }^{4}$
}

As feiras livres são consideradas o ambiente ideal para obtenção de alimentos saudáveis de qualidade, e os feirantes, por apresentarem acesso a estes alimentos, poderiam consumir satisfatoriamente alimentos in natura. O objetivo do estudo foi caracterizar os feirantes no município de Dourados, Mato Grosso do Sul, Brasil quanto às questões sociodemográficas e econômicas, o estado nutricional e o consumo alimentar. Foi aplicado questionário para obtenção das informações sociodemográficas, econômicas e de consumo alimentar. O estado nutricional foi aferido para cálculo do Índice de Massa Corporal (IMC), circunferência da cintura e Dobra Cutânea Tricipital (DCT). A comparação entre proporções foi realizada por meio da adoção do teste de Qui-quadrado e teste exato de Fisher (valor de $p \leq 0,05$ foi considerado significativo). Foram entrevistadas 74 pessoas, desse total 16 eram feirantes orgânicos e 58 convencionais, 56,8\% eram produtores, $12,2 \%$ produtores/atravessadores, $64,8 \%$ foram classificados com excesso de peso pelo IMC, sendo a obesidade mais prevalentes em mulheres obesidade $(p \leq 0,05), 58,8 \%$ apresentaram risco para doenças cardiovasculares (medida pela circunferência da cintura) e a maioria apresentou obesidade pela medida da DCT. Quanto ao consumo alimentar, os alimentos saudáveis foram mais consumidos diariamente do que os não saudáveis. Os resultados demonstraram elevada prevalência de excesso de peso e, embora o consumo de alimentos marcadores de alimentação saudável pelos feirantes tenha sido elevado, o consumo de alimentos não saudáveis foi acima do esperado.

Palavras-chave: Produção de alimentos; Estado nutricional; Consumo alimentar.

\section{Food consumption and nutritional status of market vendors of the city of Dourados - Mato Grosso do Sul, Brazil}

Open markets are considered the ideal environment for obtaining healthy quality food, and marketers, having access to these foods, could consume fresh food satisfactorily. The objective of the study was to characterize markets vendors in the minicipality of Dourados, Mato Grosso do Sul, Brazil regarding socio-demographic and economic issues, nutritional status and food consumption. A questionnaire was applied to obtain sociodemographic, economic and food consumption information. Nutritional status was measured to calculate the Body Mass Index (BMI), waist circumference and tricipital skinfold thickness (TST). The comparison between

1 Universidade Federal da Grande Dourados. Endereço para Correspondência: Rua 04 de Julho, Fátima do Sul, Mato Grosso do Sul, Brasil, Tel.: (67) 999016056. E-mail: valeria.reis88@hotmail.com. ID ORCID: https://orcid.org/0000-0002-7109-023)

2 ID ORCID: https://orcid.org/0000-0002-8517-6691

${ }^{3}$ Docente da Universidade Federal da Grande Dourados (UFGD), Dourados, Brasil. ID ORCID: https://orcid.org/0000-0002-5847-2831

${ }^{4}$ Docente da Universidade Federal da Grande Dourados (UFGD). ID ORCID: https://orcid.org/0000-0001-7340-1727 
proportions was performed using the Chi-square test and Fisher's exact test (a value of $p \leq 0.05$ was considered significant). Seventy-four people were interviewed, of which 16 were organic and 58 conventional marketers, $56.8 \%$ were producers, $12.2 \%$ were producer/middlemen, $64.8 \%$ were classified as overweight by BMI, with obesity being more prevalent in woman $(\phi \leq 0,05), 58.8 \%$ were at risk for cardiovascular diseases (measured by waist circumference) and most of them presented obesity by the TST measurement. As food consumption, healthy foods were consumed more daily than unhealthy ones. The results demonstrate a high prevalence of overweight and, although the consumption of foods that are indicative of healthy eating by marketers was high, the consumption of unhealthy food was higher than expectations.

Keywords: Food production; Nutritional status; Food consumption.

Submetido em: 02/04/2020

Aceito em: 13/04/2021

\section{INTRODUÇÃO}

As feiras livres são consideradas o ambiente ideal para obter produtos in natura, minimamente processados e frescos. A maioria das feiras ocorre em locais abertos e são utilizadas por pequenos produtores rurais, ou até mesmo atravessadores, para comercializarem seus produtos, que são constituídos, em grande parte, de alimentos hortifrutigranjeiros, cultivados em suas próprias terras ou comprados de outros fornecedores ${ }^{[1]}$.

Os alimentos comercializados em feiras são no geral in natura e considerados saudáveis, pois sofrem modificação mínima desde a colheita até a comercialização ${ }^{[2]}$. Entretanto, em relação ao teor de agrotóxicos, podem ou não conter quantidades exacerbadas, dependendo da forma de produção. Rocha et al. (2010) ${ }^{[3]}$ defendem que os alimentos das feiras apresentam pequena ou nenhuma quantidade de agrotóxicos, enquanto Luz et al. (2015) ${ }^{[4]}$ afirmam que, muito embora produtos vendidos em feiras possam ser provenientes da agricultura familiar, esses alimentos podem estar contaminados com diversos tipos de agrotóxicos.

Por produzirem ou terem fácil acesso a alimentos in natura e minimamente processados, os pequenos agricultores e os atravessadores poderiam (ou deveriam) apresentar uma alimentação saudável, rica em frutas, verduras e legumes. Entretanto, quando se faz uma análise dos inquéritos alimentares populacionais brasileiros, percebe-se que, tanto em áreas urbanas quanto rurais, meio no qual os feirantes podem viver, o consumo alimentar vem mudando consideravelmente e o aumento da ingestão de ultraprocessados em detrimentos a alimentos in natura tem crescido ${ }^{[5,6]}$.

A publicação mais recente da POF 2017/2018[6] revelou existência de maior disponibilidade de alimentos in natura ou minimamente processados nos lares brasileiros quando comparado com as pesquisas anteriores, porém a Pesquisa salienta que esses alimentos estão perdendo lugar para produtos processados e ultraprocessados com predominância de ultraprocessados $(19,8 \%)$ e processados $(11,3 \%)$ no meio urbano quando comparado ao meio rural (10,7\% e $5,5 \%$, respectivamente).

O estado nutricional do brasileiro tem variado ao longo dos anos, com aumento expressivo do excesso de peso entre diferentes classes sociais. Segundo dados do estudo "Vigilância de Fatores de Risco para doenças crônicas não transmissíveis" Vigitel de 2019, 55,4\% dos adultos apresentam sobrepeso e $20,3 \%$ obesidade[7].

Considerando a carência de estudos que revelam a qualidade da alimentação e do estado nutricional daqueles que possuem maior acesso a alimentos in natura e minimamente processados, esse trabalho visa caracterizar os feirantes no município de Dourados, Mato Grosso do Sul (MS. Brasil), quanto às questões sociodemográficas e econômicas, o estado nutricional e o consumo alimentar. 


\section{MÉTODOS}

Foi realizado um estudo transversal quantitativo, com amostragem por conveniência de feirantes que atuam nas feiras livres de Dourados/MS, no período de maio a setembro de 2017. Dourados é o segundo maior município do estado e é localizado na região centro-sul do Mato Grosso do Sul e possui uma população estimada de 222.949 habitantes ${ }^{[8]}$. O município conta com uma feira orgânica e algumas feiras convencionais em diversas localidades e apresentava, em 2017, 228 feirantes cadastrados na Secretaria Municipal de Agricultura Familiar.

Para realização da pesquisa, foram convidados a participar todos os feirantes orgânicos e convencionais maiores de 18 anos que se consideravam responsáveis pela venda e que comercializavam hortifrutigranjeiros nas principais feiras de Dourados. Foram excluídos da amostra os feirantes que comercializavam itens não alimentícios, tais como artesanatos, roupas e brinquedos, ou alimentos prontos e refeições como: pastéis, outras frituras, salgados, alimentos não hortifrutigranjeiros, restando ao final um total de 100 feirantes com estas características.

Os feirantes foram entrevistados no próprio local de trabalho, no horário de funcionamento das feiras por entrevistadores previamente treinados e da área de Nutrição. Foi aplicado para o adulto responsável pela barraca um questionário estruturado, contendo questões socioeconômicas e demográfica como: sexo, idade, raça/cor, se é agricultor ou atravessador, renda, lugar onde reside, escolaridade e número de pessoas no domicílio.

Foi também aplicado um módulo para avaliação do consumo alimentar por meio de questionário de frequência alimentar, adaptado do questionário utilizado na pesquisa Vigitel[7]. Essa avaliação conteve perguntas sobre a frequência semanal do consumo de alguns grupos de alimentos questionando a frequência nos últimos sete dias (frequência semanal para todos os alimentos). Alguns dos grupos e alimentos avaliados foram: verduras ou legumes crus, verduras ou legumes cozidos, feijão, suco artificial, doces/sobremesas, embutidos, biscoito/bolacha, salgadinhos de pacote, refrigerante e carne vermelha. Todos esses alimentos foram separados posteriormente como marcadores de alimentação saudável e marcadores de alimentação não saudável. Para marcadores de alimentação saudável foi considerada a ingestão de cinco ou mais vezes na semana de frutas, hortaliças e feijão. Marcadores de alimentação não saudável seguiram o mesmo indicado pela Pesquisa Nacional de Saúde do Escolar - PeNSE (2013), em que foi aceito o consumo de até quatro vezes na semana, sendo problemática a ingestão de cinco ou mais vezes para doces, embutidos, frituras, bolachas, salgadinhos de pacote, carne vermelha, refrigerante e suco em pó[i]. Para a análise estatística, foram organizados dois grupos, a saber: hortaliças (verduras e legumes crus e cozidas); e o, grupo contendo refrigerantes e suco em pó.

Para avaliar o estado nutricional foi aferida a altura em metros (m) e o peso em quilograma $(\mathrm{kg})$ para classificação do Índice de Massa Corporal (IMC), a circunferência da cintura em centímetros (cm) para avaliação do risco para doenças cardiovasculares e de medidas de dobra cutânea tricipital em milímetros ( $\mathrm{mm}$ ).

Para a tomada do peso, foi utilizada a balança da marca Marte ${ }^{\circledR}$ com capacidade de 199,95 $\mathrm{kg}$ e precisão de 50 gramas. A balança foi colocada em um piso plano e sem desnível. Foi solicitado ao feirante que retirasse os sapatos e qualquer objeto que estivesse em seu bolso ou mão, posicionando-o no centro da balança, com a coluna ereta e a cabeça levantada. A leitura foi feita pelo pesquisador e o resultado obtido anotado em uma folha ${ }^{[10]}$.

A altura foi aferida com um estadiômetro portátil com capacidade de $213 \mathrm{~cm}$, que foi posicionado em um piso reto sem nenhum desnível. Os feirantes foram posicionados na plataforma com postura ereta, pés descalços, juntos, com os calcanhares, nádegas e ombros encostados na barra escalonada do estadiômetro e seguindo as demais orientações do Ministério da Saúde (Brasil, 2011). A barra horizontal do estadiômetro foi abaixada e apoiada sobre o topo da cabeça, fazendo uma leve compressão no cabelo do indivíduo e feita a leitura e anotação[10]. Para definir o IMC dos adultos foi utilizado o peso $(\mathrm{kg})$, a altura ao quadrado $\left(\mathrm{m}^{2}\right)$ e classificado segundo os parâmetros da Organizaçãa Mundial da Saúde - OMS (1998)[11]. 
Para aferir a circunferência da cintura foi utilizada uma fita métrica flexível da marca Sanny ${ }^{\circledR}$ com alcance máximo de 2 metros. A medida da circunferência considerada foi o ponto médio entre a última costela e a crista ilíaca. A leitura foi feita após o feirante fazer a expiração e anotado em seguida na folha de protocolo individual[10].

A circunferência da cintura foi classificada segundo a WHO (2000) considerado para homens: maior ou igual a $94 \mathrm{~cm}$ - risco alto; e maior ou igual a $102 \mathrm{~cm}$ - risco muito alto para complicações metabólicas. Para as mulheres, foi considerado: maior ou igual a $80 \mathrm{~cm}$ - risco alto; e maior que 88 risco muito alto para complicações metabólicas ${ }^{[12]}$.

A Dobra Cutânea Tricipital (DCT) foi classificada segundo os parâmetros utilizados por Blackburn e Thornton (1979), em que menor que 90\% é desnutrição; 90 a 100\% eutrofia; 110 a 120\% sobrepeso; e acima de $120 \%$ obesidade ${ }^{[13]}$. A DCT foi aferida utilizando um adipômetro da marca Sanny ${ }^{\circledR}$ com a resolução de medida de $0,1 \mathrm{~mm}$. A prega escolhida foi a do hemicorpo direito, em seguida destacada a dobra cutânea colocando os dedos polegar e indicador posicionados cerca de $8 \mathrm{~cm}$ distante um do outro. O equipamento foi ajustado cerca de $1 \mathrm{~cm}$ do ponto anatômico. Foram realizadas três medidas não consecutivas, e, se a diferença entre os valores obtidos foi maior que $5 \%$, as medidas de DCT foram refeitas. Posteriormente foi calculada a média dos valores adquirido e obtido ao final um valor único ${ }^{[14]}$.

Os dados colhidos foram organizados em banco de dados utilizando-se o programa Excel versão 2013. A análise foi realizada com auxílio do software SPSS versão 19.

Para descrição e interpretação dos dados, o IMC foi apresentado em quatro categorias: baixo peso, eutrofia, sobrepeso e obesidade. Para a Circunferência da Cintura (CC) foi apresentado o risco alto e risco muito alto, sendo os participantes que não se apresentaram com risco alto ou muito alto foram nomeados de sem risco alto; e a DCT em desnutrição, eutrofia, sobrepeso e obesidade.

Foi elaborada análise exploratória descritiva incluindo proporções para as variáveis categóricas e medidas de dispersão e tendência central para as variáveis numéricas. A comparação entre proporções foi avaliada por meio do teste de Qui-quadrado de Pearson ou do teste exato de Fisher (quando caselas apresentaram valores inferiores a cinco) que foram consideradas significativas quando $p$-valor $\leq 0,05$. Foram testadas associações entre as variáveis de estado nutricional e de consumo alimentar com variáveis sociodemográficas (idade, sexo), econômica (renda), além do tipo de feira (orgânica ou convencional) e produção ou não de alimentos.

Este projeto é parte de um projeto maior da Faculdade de Ciências da Saúde que foi aprovado pelo Comitê de Ética em Pesquisa da Universidade Federal da Grande Dourados, com parecer de número CAAE 71689417.8.0000.5160.

\section{RESULTADOS}

\section{Condições socioeconômicas e demográficas dos feirantes}

Dos 100 feirantes cadastrados que seguiam o critério de inclusão, 74 concederam a entrevista assinando o Termo de Consentimento Livre e Esclarecido. Os demais não quiseram participar da pesquisa. Dos 74 feirantes, 16 atuavam na feira orgânica do município e os demais às feiras convencionais.

A Tabela 1 mostra que um pouco mais da metade dos entrevistados eram do sexo masculino, com idade média de 50,4 anos (Desvio Padrão - DP $=14,6)$. Quase metade dos feirantes se autodeclararam de cor preta/parda $(48,6 \%)$ seguida da branca $(35,1 \%)$. A média de escolaridade entre os entrevistados foi de 8,8 anos $(\mathrm{DP}=4,2)$ e a renda familiar per capita em sua maioria foi $\geq 1 / 2$ a 2 salários mínimos (SM). Metade dos feirantes residiam em Dourados, em casas próprias.

Mais da metade dos entrevistados se auto declarou agricultores familiares com alimentos oriundos da própria roça/lavoura. Dos que se declaram atravessadores, a procedência dos alimentos citadas foram depósitos de hortifrúti da cidade de Dourados, Campo Grande, do estado de Minas Gerais ou São Paulo, e a compra direta de outros agricultores. Apenas um indígena e um 
feirante que se autodeclarou de cor preta/parda recebia o benefício do Programa Bolsa Família.

Em relação às perdas de dados, dos 74 entrevistados, dois feirantes se recusaram a informar a renda familiar total, outros quatro feirantes não foram localizados novamente para aferir o peso corporal e apenas um feirante se recusou a realizar as medidas antropométricas.

Tabela 1. Perfil demográfico e socioeconômico de feirantes do município de Dourados-MS, Brasil, 2017.

\begin{tabular}{|c|c|c|}
\hline Variáveis & n & $\%$ \\
\hline \multicolumn{3}{|l|}{ Sexo } \\
\hline Masculino & 39 & 52,7 \\
\hline Feminino & 35 & 47,3 \\
\hline \multicolumn{3}{|l|}{ Faixa etária (anos) } \\
\hline $19-39$ & 17 & 23,0 \\
\hline $40-59$ & 37 & 50,0 \\
\hline$>60$ & 20 & 27,0 \\
\hline \multicolumn{3}{|l|}{ Cor/Raça autodeclarada } \\
\hline Branca & 26 & 35,1 \\
\hline Preta/Parda & 36 & 48,6 \\
\hline Amarela & 10 & 13,5 \\
\hline Indígena & 2 & 2,7 \\
\hline \multicolumn{3}{|l|}{ Escolaridade (em anos) } \\
\hline 0 a 4 & 12 & 16,2 \\
\hline 5 a 7 & 10 & 13,5 \\
\hline 8 ou + & 52 & 70,3 \\
\hline \multicolumn{3}{|l|}{ Renda (per capita) } \\
\hline$<1 / 2$ salário mínimo & 24 & 32,4 \\
\hline$>1 / 2$ a 2 salários mínimos & 43 & 58,1 \\
\hline >2 salários mínimos & 5 & 6,8 \\
\hline Não sabe/Não respondeu & 2 & 2,7 \\
\hline \multicolumn{3}{|l|}{ Local de residência } \\
\hline Dourados & 57 & 77,0 \\
\hline Outro município & 17 & 23,0 \\
\hline \multicolumn{3}{|l|}{ Condições da moradia } \\
\hline Próprio & 62 & 83,8 \\
\hline Alugado & 2 & 2,7 \\
\hline Cedido & 5 & 6,8 \\
\hline Assentado & 5 & 6,8 \\
\hline \multicolumn{3}{|c|}{ Número de pessoas no domicílio } \\
\hline 1 a 3 & 44 & 59,5 \\
\hline 4 a 6 & 29 & 39,2 \\
\hline $7 \mathrm{ou}+$ & 1 & 1,4 \\
\hline \multicolumn{3}{|l|}{ É agricultor } \\
\hline $\operatorname{Sim}$ & 42 & 56,8 \\
\hline Não & 32 & 43,2 \\
\hline É produtor e atravessador & 9 & 12,2 \\
\hline \multicolumn{3}{|c|}{ De onde vem os produtos vendidos na feira } \\
\hline Da sua roça/lavoura & 41 & 55,4 \\
\hline CEASA* & 4 & 5,4 \\
\hline De outro local & 37 & 50,0 \\
\hline
\end{tabular}


Caracterização do consumo alimentar e estado nutricional de feirantes. Narciso et al..

\begin{tabular}{lrr} 
Recebe auxílio Bolsa Família & 2 & 2,7 \\
Sim & 67 & 90,5 \\
Não & 5 & 6,8 \\
Não sabe/Não respondeu & \\
\hline
\end{tabular}

Legenda: CEASA: Centrais de Abastecimento

\section{Estado nutricional dos feirantes}

A maioria dos feirantes $(64,8 \%)$ foi classificada com excesso de peso (sobrepeso e obesidade), segundo o IMC. Para a CC, 58,1\% dos entrevistados apresentaram algum risco para doença cardiovascular e, segundo a DCT, 55,4\% apresentaram obesidade. Entretanto, foi observado que aproximadamente um quarto dos indivíduos apresentou baixo peso, proproção que não foi encontrada quando considerado qualquer outro parâmetro adotado. (Gráfico 1).

A Tabela 2 apresenta a relação entre o estado nutricional e as variáveis sociodemográficas, econômicas, tipo de feira e produção ou não de alimentos entre feirantes. Em relação a associação do estado nutricional com as variáveis sociodemográficas, observou-se que entre aqueles que tinham sobrepeso, 55,6\% eram homens e 44,4\% eram mulheres. Entre os obesos, a maior proporção era composta por mulheres $(83,3 \%$; $p$-valor $\leq 0,05)$. A respeito da associação entre a CC e as variáveis sociodemográficas e econômicas, resultados similares ao do IMC foram encontrados. Entre aqueles que apresentam alto risco, 58,8\% eram do sexo masculino. Já entre os indivíduos que apresentam risco muito alto, $75,0 \%$ eram do sexo feminino ( $p$-valor $\leq 0,05)$. As demais variáveis estudadas não foram estatisticamente associadas ao estado nutricional investigado pelo IMC e CC. Quanto ao estado nutricional obtido pela DCT, não houve foi observada associação significativa com nenhuma das variáveis estudadas (Tabela 2).

Gráfico 1. Estado Nutricional avaliado por meio do Índice de Massa Corporal (IMC), Circunferência da Cintura (CC) e Dobra Cutânea Tricipital (DCT) de feirantes do município de Dourados-MS, Brasil, 2017.

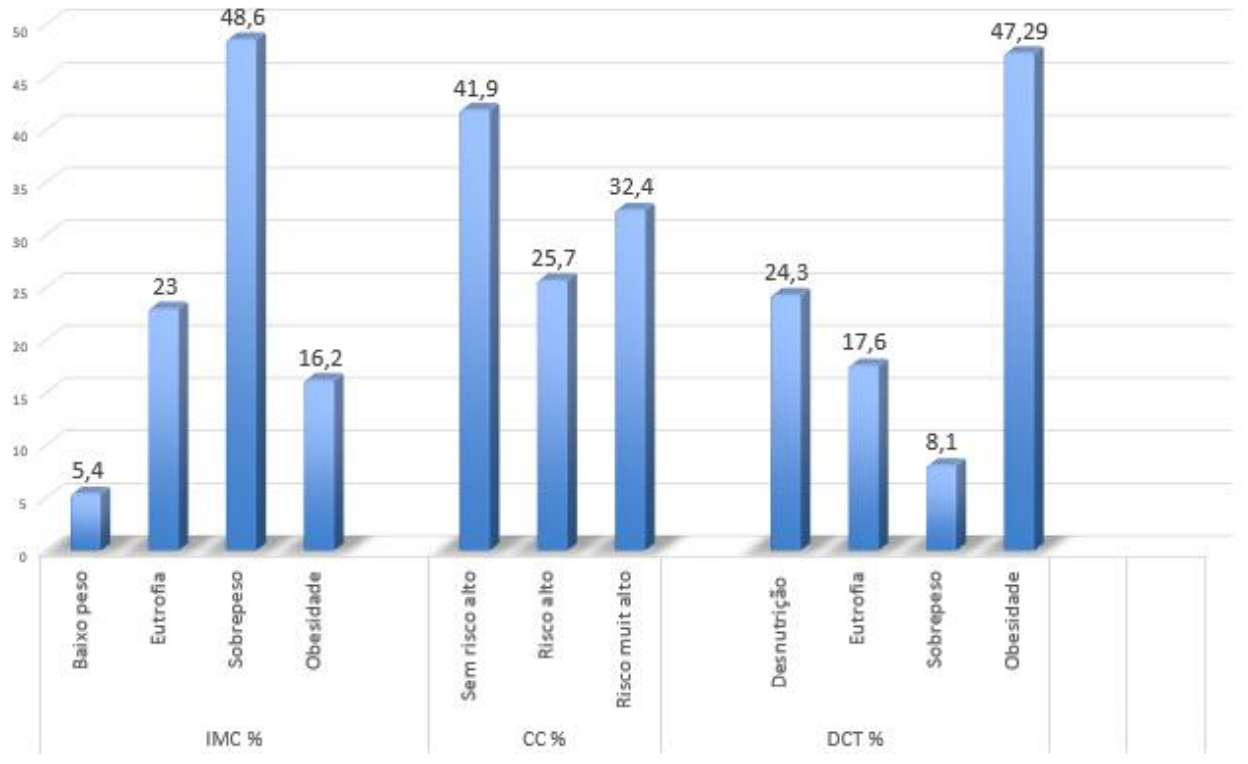


Tabela 2. Estado nutricional em relação a variáveis sociodemográficas, tipo de feira e produção ou não de alimentos entre feirantes de Dourados-MS, 2017.

\begin{tabular}{|c|c|c|c|c|c|c|c|c|c|c|c|c|c|c|c|c|c|c|c|c|c|c|}
\hline \multirow[t]{4}{*}{ Variável } & \multicolumn{22}{|c|}{ Estado N utricional } \\
\hline & \multicolumn{8}{|c|}{ Indce de Massa Corpora } & \multicolumn{6}{|c|}{ Circunferência da Cintura } & \multicolumn{8}{|c|}{ Dobra Cutânea Tricipital } \\
\hline & \multicolumn{2}{|c|}{ Baixo peso } & \multicolumn{2}{|c|}{ Eutrofia } & \multicolumn{2}{|c|}{ Sobrepeso } & \multicolumn{2}{|c|}{ Obesidade } & \multicolumn{2}{|c|}{ Sem risco } & \multicolumn{2}{|c|}{ Risco alto } & \multicolumn{2}{|c|}{ Risco muito alt } & \multicolumn{2}{|c|}{ Desnutrição } & \multicolumn{2}{|c|}{ Eutrofia } & \multicolumn{2}{|c|}{ Sobrepeso } & \multicolumn{2}{|c|}{ Obesidade } \\
\hline & $\mathrm{n}$ & $\%$ & $\mathrm{n}$ & $\%$ & $\mathrm{n}$ & $\%$ & $\mathrm{n}$ & $\%$ & $\mathrm{n}$ & $\%$ & $\mathrm{n}$ & $\%$ & $\mathrm{n}$ & $\%$ & $\mathrm{n}$ & $\%$ & $\mathrm{n}$ & $\%$ & $\mathrm{n}$ & $\%$ & $\mathrm{n}$ & $\%$ \\
\hline \multicolumn{23}{|l|}{ Idade } \\
\hline 19 a 39 anos & 1,0 & 25,0 & 1,0 & 5,9 & 10,0 & 27,8 & 2,0 & 16,7 & 8,0 & 25,8 & 4,0 & 23,5 & 4,0 & 16,7 & 2,0 & 11,1 & 1,0 & 7,7 & 3,0 & 50,0 & 10,0 & 28,6 \\
\hline 40 a 59 anos & 2,0 & 50,0 & 10,0 & 58,8 & 15,0 & 41,7 & 9,0 & 75,0 & 18,0 & 58,1 & 8,0 & 47,1 & 11,0 & 45,8 & 10,0 & 55,6 & 6,0 & 46,2 & 2,0 & 33,3 & 19,0 & 54,3 \\
\hline $60 \mathrm{e}+$ anos & 1,0 & 25,0 & 6,0 & 35,3 & 11,0 & 30,6 & 1,0 & 8,3 & 5,0 & 16,1 & 5,0 & 29,4 & 9,0 & 37,5 & 6,0 & 33,3 & 6,0 & 46,2 & 1,0 & 16,7 & 6,0 & 17,1 \\
\hline Masculino & 2,0 & 50,0 & 13,0 & 76,5 & 20,0 & 55,6 & 2,0 & 16,7 & 22,0 & 71,0 & 10,0 & 58,8 & 6,0 & 25,0 & 10,0 & 55,6 & 5,0 & 38,5 & 1,0 & 16,7 & 22,0 & 62,9 \\
\hline Feminino & 2,0 & 50,0 & 4,0 & 23,5 & 16,0 & 44,4 & 10,0 & 83,3 & 9,0 & 29 & 7,0 & 41,2 & 18,0 & 75,0 & 8,0 & 44,4 & 8,0 & 61,5 & 5,0 & 83,3 & 13,0 & 37,1 \\
\hline \multicolumn{23}{|l|}{ Renda } \\
\hline$\leq 1 / 2 \mathrm{SM}$ & 1,0 & 25,0 & 4,0 & 23,5 & 15,0 & 44,1 & 2,0 & 16,7 & 5,0 & 16,7 & 6,0 & 35,3 & 11,0 & 47,8 & 6,0 & 33,3 & 4,0 & 33,3 & 4,0 & 66,7 & 8,0 & 23,5 \\
\hline$>1 / 2$ a $2 S M$ & 3,0 & 75,0 & 11,0 & 64,7 & 18,0 & 52,9 & 8,0 & 66,7 & 24,0 & 80,0 & 9,0 & 52,9 & 10,0 & 43,5 & 11,0 & 61,1 & 6,0 & 50,0 & 2,0 & 33,3 & 24,0 & 70,6 \\
\hline$>2 \mathrm{SM}$ & 0,0 & 0,0 & 2,0 & 11,8 & 1,0 & 2,9 & 2,0 & 16,7 & 1,0 & 3,3 & 2,0 & 11,8 & 2,0 & 8,7 & 1,0 & 5,6 & 2,0 & 16,7 & 0,0 & 0,0 & 2,0 & 5,9 \\
\hline \multicolumn{23}{|l|}{ Tipo de Feira } \\
\hline Orgânica & 1,0 & 25,0 & 3,0 & 17,6 & 6,0 & 16,7 & 4,0 & 33,3 & 5,0 & 16,1 & 2,0 & 11,8 & 8,0 & 33,3 & 4,0 & 22,2 & 3,0 & 23,1 & 1,0 & 16,7 & 7,0 & 20,0 \\
\hline Não orgânica & 3,0 & 75,0 & 14,0 & 82,4 & 30,0 & 83,3 & 8,0 & 66,7 & 26,0 & 83,9 & 15,0 & 88,2 & 16,0 & 66,7 & 14,0 & 77,8 & 10,0 & 76,9 & 5,0 & 83,3 & 28,0 & 80,0 \\
\hline \multicolumn{23}{|c|}{ Agricultor/ Produtor } \\
\hline Sim & 2,0 & 50,0 & 8,0 & 47,1 & 22,0 & 61,1 & 7,0 & 58,3 & 14,0 & 45,2 & 9,0 & 52,9 & 17,0 & 79,8 & 8,0 & 44,4 & 10,0 & 76,9 & 3,0 & 50,0 & 19,0 & 54,3 \\
\hline Não & 2,0 & 50,0 & 9,0 & 52,9 & 14,0 & 38,9 & 5,0 & 41,7 & 17,0 & 54,8 & 8,0 & 47,1 & 7,0 & 29,2 & 10,0 & 55,6 & 3,0 & 23,1 & 3,0 & 50,0 & 16,0 & 45,7 \\
\hline
\end{tabular}

SM: Salário Mínimo (em 2017 no valor de R\$ 937,00)

Em negrito: associações com significância estatística ( $p$-valor $\leq 0,05$ ).

N ota: Não foi aplicado teste estatístico para variáveis com caselas que apresentam valor "zero" 


\section{Consumo alimentar dos feirantes}

Em relação a associação dos marcadores de alimentação saudável com as variáveis sociodemográficas, a prevalência de consumo de hortaliças foi igual ou maior que cinco dias na semanas, foi mais elevada entre indivíduos com idade entre 40 e 59 anos $(54,8 \%)$ quando comparada a adultos jovens $(17,7 \%)$ e a idosos $(27,4 \%)$ ( $p$-valor $\leq 0,05)$, sendo o consumo de frutas maior entre os idosos (dados sem significância estatística) (Tabela $3)$.

A respeito da relação entre as variáveis sociodemográficas e os marcadores de alimentação não saudável, observou-se maior frequência de consumo de bolacha, em cinco ou mais dias na semana, entre os indivíduos integrantes da faixa etária de 19 a 39 anos (60,0\%), quando comparado às faixas etárias superiores e de carne vermelha entre homens $(67,7 \%)$ do que entre mulheres $(32,3 \%$; $p$ valor $\leq 0,05)$. As demais variáveis sociodemográficas e econômica não foram significativamente associadas com consumo de marcadores saudáveis e não saudáveis (Tabela 3). Os resultados apresentados na Tabela 4 sugerem que ser feirante de feiras orgânicas ou convencionais e produzir ou não os alimentos não interferiram, de forma significativa, no consumo de marcadores de alimentação saudável e não saudável ( $p$-valor $>0,05)$. 
Tabela 3. Consumo alimentar de marcadores de alimentação saudável e não saudável em relação a variáveis sociodemográficas de feirantes de Dourados-MS, Brasil, 2017.

\begin{tabular}{|c|c|c|c|c|c|c|c|c|c|c|c|c|c|c|c|c|}
\hline \multirow[t]{3}{*}{ Variável } & \multicolumn{6}{|c|}{ Idade } & \multicolumn{4}{|c|}{ Sexo } & \multicolumn{6}{|c|}{ Renda } \\
\hline & \multicolumn{2}{|c|}{19 a 39 anos } & \multicolumn{2}{|c|}{40 a 59 anos } & \multicolumn{2}{|c|}{$\geq 60$ anos } & \multicolumn{2}{|c|}{ Masculino } & \multicolumn{2}{|c|}{ Feminino } & \multicolumn{2}{|c|}{$\leq 1 / 2 S M$} & \multicolumn{2}{|c|}{$>1 / 2$ a $2 S M$} & \multicolumn{2}{|c|}{$>2 \mathrm{SM}$} \\
\hline & $\mathrm{n}$ & $\%$ & $\mathrm{n}$ & $\%$ & $\mathrm{n}$ & $\%$ & $\mathrm{n}$ & $\%$ & $\mathrm{n}$ & $\%$ & $\mathrm{n}$ & $\%$ & $\mathrm{n}$ & $\%$ & $\mathrm{n}$ & $\%$ \\
\hline \multicolumn{17}{|c|}{$\begin{array}{l}\text { Marcadores de Alimentação Saudável } \\
\text { Fruta }\end{array}$} \\
\hline$\geq 5 \mathrm{x} /$ semana & 10,0 & 20,0 & 25 & 10,0 & 15 & 30,0 & 24 & 48,0 & 26 & 52,0 & 19 & 39,6 & 25 & 52,1 & 4 & 8,3 \\
\hline $\begin{array}{l}<5 \mathrm{x} / \text { semana } \\
\text { H ortaliça }\end{array}$ & 7 & 29,2 & 12 & 7,0 & 20 & 20,8 & 15 & 62,5 & 9 & 37,5 & 5 & 20,8 & 18 & 75,0 & 1 & 4,2 \\
\hline$\geq 5 \mathrm{x} /$ semana & 11 & 17,7 & 34 & 54,8 & 17 & 27,4 & 35 & 56,5 & 27 & 43,5 & 19 & 31,7 & 37 & 61,7 & 4 & 6,7 \\
\hline$<5 \mathrm{x} /$ semana & 6 & 50,0 & 3 & 25,0 & 3 & 25,0 & 4 & 33,3 & 8 & 66,7 & 5 & 41,7 & 6 & 50,0 & 1 & 8,3 \\
\hline \multicolumn{17}{|l|}{ Feijão } \\
\hline$\geq 5 \mathrm{x} /$ semana & 13 & 24,1 & 23 & 42,6 & 18 & 33,3 & 31 & 57,4 & 23 & 42,6 & 20 & 38,5 & 29 & 55,8 & 3 & 5,8 \\
\hline$<5 \mathrm{x} /$ semana & 4 & 20,0 & 14 & 70,0 & 2 & 10,0 & 8 & 40,0 & 12 & 60,0 & 4 & 20,0 & 14 & 70,0 & 2 & 10,0 \\
\hline \multicolumn{17}{|c|}{$\begin{array}{l}\text { Marcadores de Alimentação N ão Saudável } \\
\text { Doces }\end{array}$} \\
\hline$\geq 5 \mathrm{x} /$ semana & 5 & 24,4 & 7 & 41,2 & 5 & 29,4 & 9 & 52,9 & 8 & 47,1 & 5 & 31,3 & 10 & 62,5 & 1 & 6,3 \\
\hline$<5 \mathrm{x} /$ semana & 12 & 21,1 & 30 & 52,6 & 15 & 26,3 & 30 & 52,6 & 27 & 47,4 & 19 & 33,9 & 33 & 58,9 & 4 & 7,1 \\
\hline \multicolumn{17}{|l|}{ Embutidos } \\
\hline$\geq 5 \mathrm{x} /$ semana & 2 & 40,0 & 2 & 40,0 & 1 & 20,0 & 5 & 100,0 & 0 & 0,0 & 2 & 50,0 & 2 & 50,0 & 0 & 0,0 \\
\hline \multicolumn{17}{|l|}{ Frituras } \\
\hline$\geq 5 \mathrm{x} /$ semana & 2 & 40,0 & 2 & 40,0 & 1 & 20,0 & 4 & 80,0 & 1 & 20,0 & 0 & 0,0 & 4 & 100,0 & 0 & 0,0 \\
\hline$<5 \mathrm{x} /$ semana & 15 & 21,7 & 35 & 50,7 & 19 & 27,5 & 35 & 50,7 & 34 & 49,3 & 24 & 35,3 & 39 & 57,4 & 5 & 7,4 \\
\hline \multicolumn{17}{|l|}{ Bolacha } \\
\hline$\geq 5 \mathrm{x} /$ semana & 6 & 60,0 & 1 & 10,0 & 3 & 30,0 & 4 & 40,0 & 6 & 60,0 & 5 & 62,5 & 3 & 37,5 & 0 & 0,0 \\
\hline$<5 \mathrm{x} /$ semana & 11 & 17,2 & 36 & 56,3 & 17 & 26,6 & 35 & 54,7 & 29 & 45,3 & 19 & 29,7 & 40 & 62,5 & 5 & 7,8 \\
\hline \multicolumn{17}{|c|}{ Salgadinho de pacote } \\
\hline$\geq 5 \mathrm{x} /$ semana & 4 & 66,7 & 2 & 33,3 & 0 & 0,0 & 4 & 66,7 & 2 & 33,3 & 2 & 40,0 & 3 & 60,0 & 0 & 0,0 \\
\hline$<5 \mathrm{x} /$ semana & 13 & 19,1 & 35 & 51,5 & 20 & 29,4 & 35 & 51,5 & 33 & 48,5 & 22 & 32,8 & 40 & 59,7 & 5 & 7,5 \\
\hline \multicolumn{17}{|c|}{ Carne Vermelha } \\
\hline$\geq 5 \mathrm{x} /$ semana & 7 & 22,6 & 14 & 45,2 & 10 & 32,3 & 21 & 67,7 & 10 & 32,3 & 8 & 26,7 & 19 & 63,3 & 3,0 & 10,0 \\
\hline$<5 \mathrm{x} /$ semana & 10 & 23,3 & 23 & 53,5 & 10 & 23,3 & 18 & 41,9 & 25 & 58,1 & 16 & 38,1 & 24 & 57,1 & 2,0 & 4,8 \\
\hline Refrigerante $\mathrm{e}$ & & & & & & & & & & & & & & & & \\
\hline$\geq 5 x /$ semana & 7 & 38,9 & 7 & 38,9 & 4 & 22,2 & 9 & 50,0 & 9 & 50,0 & 6 & 37,5 & 9 & 56,3 & 1,0 & 6,3 \\
\hline$<5 \mathrm{x} /$ semana & 10 & 17,9 & 30 & 53,6 & 16 & 28,6 & 30 & 53,6 & 26 & 46,4 & 18 & 32,1 & 34 & 60,7 & 4,0 & 7,1 \\
\hline
\end{tabular}

SM: Salário Mínimo (em 2017 no valor de $\mathbf{R} \$ 937,00)$

Em negrito: associações com significância estatística ( $p$-valor $\leq 0,05)$.

Nota: Não foi aplicado teste estatístico para variáveis com caselas que apresentam valor "zero" 
Tabela 4. Consuo alimentar de marcadores de alimentação saudável e não saudável em relação ao tipo de feira e produção ou não de alimentos em feirantes de Dourados-MS, Brasil, 2017

\begin{tabular}{|c|c|c|c|c|c|c|c|c|}
\hline \multirow[t]{3}{*}{ Variável } & \multicolumn{4}{|c|}{$\begin{array}{c}\text { Tipo de Feira } \\
\end{array}$} & \multicolumn{4}{|c|}{ Agricultor } \\
\hline & \multicolumn{2}{|c|}{ Orgânica } & \multicolumn{2}{|c|}{ Não orgânica } & \multicolumn{2}{|c|}{ Sim } & \multicolumn{2}{|c|}{ Não } \\
\hline & $\mathrm{n}$ & $\%$ & $\mathrm{n}$ & $\%$ & $\mathrm{n}$ & $\%$ & $\mathrm{n}$ & $\%$ \\
\hline \multicolumn{9}{|c|}{$\begin{array}{l}\text { Marcadores de Alimentação Saudável } \\
\text { Fruta }\end{array}$} \\
\hline$\geq 5 x /$ semana & 11 & 22,0 & 39 & 78,0 & 28 & 56,0 & 22 & 44,0 \\
\hline$<5 \mathrm{x} /$ semana & 5 & 20,8 & 19 & 79,2 & 14 & 58,3 & 10 & 41,7 \\
\hline \multicolumn{9}{|l|}{ H ortaliça } \\
\hline$\geq 5 x /$ semana & 11 & 17,7 & 51 & 82,3 & 34 & 54,8 & 28 & 45,2 \\
\hline$<5 \mathrm{x} /$ semana & 5 & 41,7 & 7 & 58,3 & 8 & 66,7 & 4 & 33,3 \\
\hline \multicolumn{9}{|l|}{ Feijão } \\
\hline$\geq 5 \mathrm{x} /$ semana & 10 & 18,5 & 44 & 81,5 & 32 & 59,3 & 22 & 40,7 \\
\hline$<5 \mathrm{x} /$ semana & 6 & 30,0 & 14 & 70,0 & 10 & 50,0 & 10 & 50,0 \\
\hline \multicolumn{9}{|c|}{$\begin{array}{l}\text { Marcadores de Alimentação N ão Saudável } \\
\text { Doces }\end{array}$} \\
\hline$\geq 5 x /$ semana & 4 & 23,5 & 13 & 76,5 & 9 & 52,9 & 8 & 41,1 \\
\hline$<5 \mathrm{x} /$ semana & 12 & 21,1 & 45 & 78,9 & 33 & 57,9 & 24 & 42,1 \\
\hline \multicolumn{9}{|l|}{ Embutidos } \\
\hline$\geq 5 x /$ semana & 1 & 20,0 & 4 & 80,0 & 3 & 60,0 & 2 & 40,0 \\
\hline$<5 \mathrm{x} /$ semana & 15 & 21,7 & 54 & 78,3 & 39 & 56,5 & 30 & 43,5 \\
\hline \multicolumn{9}{|l|}{ Frituras } \\
\hline$\geq 5 x /$ semana & 0 & 0,0 & 5 & 100,0 & 3 & 60,0 & 2 & 40,0 \\
\hline$<5 \mathrm{x} /$ semana & 16 & 23,2 & 53 & 76,8 & 39 & 56,5 & 30 & 43,5 \\
\hline \multicolumn{9}{|l|}{ Bolacha } \\
\hline$\geq 5 x /$ semana & 3 & 30,0 & 7 & 70,0 & 5 & 50,0 & 5 & 50,0 \\
\hline$<5 \mathrm{x} /$ semana & 13 & 20,3 & 51 & 79,7 & 37 & 57,8 & 27 & 42,2 \\
\hline \multicolumn{9}{|c|}{ Salgadinho de pacote } \\
\hline$\geq 5 \mathrm{x} /$ semana & 1 & 16,7 & 5 & 83,3 & 3 & 50,0 & 3 & 50,0 \\
\hline$<5 \mathrm{x} /$ semana & 15 & 22,1 & 53 & 77,9 & 39 & 57,4 & 29 & 42,6 \\
\hline \multicolumn{9}{|c|}{ Carne Vermelha } \\
\hline$\geq 5 \mathrm{x} /$ semana & 6 & 19,4 & 25 & 80,6 & 17 & 54,8 & 14 & 45,2 \\
\hline$<5 \mathrm{x} /$ semana & 10 & 23,3 & 33 & 76,7 & 25 & 58,1 & 18 & 41,9 \\
\hline \multicolumn{9}{|c|}{ Refrigerante e suco em pó } \\
\hline$\geq 5 \mathrm{x} /$ semana & 2 & 11,1 & 16 & 88,9 & 11 & 61,1 & 7 & 38,9 \\
\hline$<5 \mathrm{x} /$ semana & 14 & 25,0 & 42 & 75,0 & 31 & 55,4 & 25 & 44,6 \\
\hline
\end{tabular}




\section{DISCUSSÃO}

\section{Questões socioeconômicas}

No presente trabalho foi analisado o perfil dos feirantes do município de Dourados-MS. Os resultados demonstraram que, em relação ao sexo, os achados são similares aos estudos elaborados por Mascarenhas (2014) ${ }^{[15]}$ e Amor et al. (2012) ${ }^{[16]}$, em que foi constatado que a quantidade de homens e mulheres que estavam inseridos no ambiente da feira era praticamente igual, o que mostra que as mulheres vêm desempenhando papéis similares aos dos homens tanto na participação do cultivo de alimentos, como na venda dos produtos produzidos ou revendidos nas feiras.

A média de idade encontrada neste estudo foi de 50,4 anos, dados similares aos do estudo feito por Toniasso et al. (2007)[17] com agricultores familiares e assentados no município de Jaraguari, situado também no estado do Mato Grosso do Sul, em que a grande maioria estava acima dos 50 anos, sendo possível salientar que uma pequena minoria dos agricultores (aproximadamente 6,0\%) estavam entre 24 e 34 anos, muito semelhante ao encontrado neste estudo, confirmando que, em ambos os estudos, as gerações mais novas não se ocupam dessa atividade, pois provavelmente estão cada vez mais aumentando o seu grau de escolaridade e se qualificando para desempenhar outros tipos de trabalho. Este dado pode ser comprovado pelo Censo de 2010 do Instituto Brasileiro de Geografia e Estatística (IBGE) ${ }^{[18]}$.

Em relação à raça/cor, $48,6 \%$ se autodeclararam pretos ou pardos nessa pesquisa. Esse dado corrobora os resultados da Pesquisa Nacional por Amostra de Domicílio - PNAD (2019) em que houve aumento no número de brasileiros que se autodeclararam pretos ou pardos, ultrapassando $55,0 \%$ da população brasileira ${ }^{[19]}$.

A escolaridade completa citada pela grande maioria dos feirantes $(70,3 \%)$ foi de 8 anos ou mais, sendo que apenas $16,2 \%$ relataram sem estudo ou com ensino fundamental incompleto. Isto mostra que o perfil do feirante vem se modificando aos longos dos anos devido ao desemprego $e$ as dificuldades financeiras ocasionadas pelas sucessivas crises, pois anteriormente $\mathrm{O}$ perfil desses trabalhadores em sua maioria eram de analfabetos ou semianalfabetos e uma pequena parcela possuía nível superior completo, bem diferente do cenário atual, em que há presença de feirantes que possuem ensino superior completo, alguns são universitários ou possuem algum tipo de pós-graduação ${ }^{[16]}$.

Ao que tange a renda dos entrevistados, os feirantes de Dourados são o reflexo da média de salário mensal de grande parte da população brasileira. De acordo com a PNAD (2019), mais de 30 milhões de brasileiros possuíam renda menor que um salário mínimo e mais de 31 milhões recebiam de um a dois, sendo que cerca de $85,6 \%$ possuem moradia própriaa[19].

A respeito do recebimento pelo Programa Bolsa Família, a grande maioria dos feirantes relataram que não recebe o benefício. Apenas um negro e um indígena recebem. Grande parte dos indígenas de Dourados recebe auxílio de programas governamentais devido a condições precárias e subumanas de existência, assim como muitos negros ainda nesse país, que sofrem com o preconceito da sociedade e possuem mais dificuldade de se inserirem no mercado de trabalho formal, recorrendo a políticas públicas. Além disso, verifica-se que existem poucos indígenas nas feiras de Dourados, sendo que existem mais de 15.000 indivíduos pertencentes as etnias Guarani Ñandeva, Guarani Kaiowá e Terena no município ${ }^{[2]}$. Suas terras, deficientes em tamanho, são em grande parte, improdutivas e dos que conseguem produzir, pouco é excedente para a comercialização, além do problema da falta de água na reserva indígena de Dourados ${ }^{20,21]}$.

\section{Estado nutricional}

À partir dos resultados desse estudo que utilizou o IMC, CC, DCT como preditores do estado nutricional, pode-se notar que a grande maioria dos feirantes em que foi realizada a medida antropométrica, apresentou excesso de peso. Esse resultado se assemelha aos dados de inquéritos conduzidos envolvendo a população em geral, a exemplo da Pesquisa Nacional de Saúde - PNS (2013) ${ }^{[22]}$ em que a prevalência de excesso de peso da população brasileira foi de 56,9\%. Já na pesquisa realizada nas capitais do Brasil e no Distrito Federal, o inquérito Vigitel (2019) revela que $55,7 \%$ da 
população brasileira apresenta excesso de peso, demonstrando que esta condição está presente em todas as localidades brasileiras.

A pesquisa Vigitel demonstrou também que o sexo masculino obteve uma maior porcentagem de sobrepeso do que o sexo feminino, similar ao encontrado entre os feirantes homens desta pesquisa. Mas a obesidade (IMC $>30 \mathrm{Kg} / \mathrm{m} 2)$, assim como os achados desta pesquisa, foi maior entre as mulheres de quase todas as capitais brasileiras, o que demonstra que esta condição predomina no sexo feminino[7].

Os dados desta pesquisa não revelaram diferença estatística significativa quando testadas entre renda e demais variáveis sociodemográficas, provavelmente pela amostra ser reduzida, diferentemente de um estudo recente feito por Gomes et al. (2019) que usou inquérito populacional para analisar mudanças nas prevalências de obesidade entre adultos brasileiros considerando o nível de escolaridade, revelou que a relação entre condição socioeconômica ainda não é muito bem estabelecida entre países de média renda como o Brasil, mas que a escolaridade está diretamente associada à prevalência de sobrepeso e obesidade entre homens no Brasil, apontando que há uma tendência maior de excesso de peso entre homens quando o nível acadêmico é maior, porém isso é inverso quando associado entre mulheres ${ }^{[23]}$.

\section{Consumo alimentar}

Com base nos dados obtidos, foi possível perceber a idade é um fator importante na decisão sobre a qualidade da dieta, em que é comum adultos mais jovens consumirem menos hortaliças do que idosos. Dados do inquérito nacional que avalia o consumo alimentar da população brasileira, a pesquisa Vigitel (2018)[7] comprova que com o avançar da idade a preocupação com a saúde aumenta, provavelmente devido às doenças que acabam surgindo nesse período da vida.

Assim como a Vigitel de 2018)[7], o mesmo foi encontrado para o consumo de alguns alimentos ultraprocessados, como a bolacha/biscoitos, em que adultos mais jovens apresentaram maior consumo. Embora não tenha havido associação estatisticamente significativa, também foi observado menor consumo de embutidos, frituras e refrigerantes entre idosos, seguindo também o padrão nacional de menor consumo de marcadores de alimentação não saudável entre os mais velhos.

As pesquisas nacionais (POF - 2008/09) ${ }^{[5]} \mathrm{e}$ a VIGITEL (2016)[24] também apontam para o maior consumo de carnes entre os homens adultos mais velhos, bem como os achados de Ávila et al. (2016), em inquérito realizado no município de Campinas$\mathrm{SP}$, que apontou maior consumo entre homens ${ }^{[25]}$.

Estes dados são importantes pois, ainda que nem todos os dados de consumo alimentar tenham dado significativos estatisticamente, de uma forma geral, o consumo de ultraprocessados (embutidos, salgadinho de pacote, bolachas/biscoitos e refrigerantes/sucos em pó) e excesso de carnes têm aumentado, sobretudo nos mais jovens e homens. E sabe-se que esses produtos são um dos principais contribuintes para ganho de peso ao longo da vida, e que diretamente contribuem para elevação dos riscos cardiovasculares ${ }^{[2]]}$.

No presente estudo, a renda não apresentou nenhuma significância estatística quando comparada ao consumo alimentar de marcadores saudáveis e não saudáveis, diferentemente dos resultados encontrados pela Pesquisa de Orçamentos Familiares (POF 2008/09, POF 2017/18), que mostrou que o aumento da renda tem uma relação com o maior consumo de alimentos não saudáveis ${ }^{[5,6]}$.

A respeito da associação entre marcadores de alimentação saudável com as variáveis tipo de feira e se é agricultor ou não, os resultados desta pesquisa, embora não tenham apresentado associação estatística significativa, se diferem do encontrado por Oliveira (2014) ${ }^{[27]}$, em um estudo com agricultores familiares orgânicos do Ceará, em que eles relatam que a partir do momento em que começaram a produzir produtos orgânicos, aumentou o consumo de frutas e verduras em virtude da modificação do modo de plantio, além de terem a certeza de que o alimento é mais saudável. No entanto, o consumo de hortaliças, feijão e refrigerantes foi diferente dos encontrados por Alves e Boog (2008) ${ }^{[28]} \mathrm{em}$ um estudo feito com fruticultores da zona rural de Valinhos-SP, em que a maioria relataram baixo consumo de frutas, verduras, justificando que não consomem com mais frequência esses alimentos por 
acharem que eles não produzem aporte energético necessário para as atividades que exercem ao longo do dia, pois a maioria comprava alimentos considerados mais energéticos e importantes para se saciarem, como por exemplo a carne, o feijão e o arroz.

Sabe-se que o consumo frequente de frutas e hortaliças é essencial para o organismo, pois o baixo consumo desses alimentos está intimamente ligado ao surgimento de DCNT e o aumento da obesidade [2]. Por isso possuir uma alimentação saudável rica em frutas, verduras é de grande importância, assim como preconiza o Guia Alimentar para a População Brasileira, publicado em 2014, que preconiza que a base da alimentação seja de alimentos in natura e minimamente processados ${ }^{[2]}$.

\section{Limitações do estudo}

Uma limitação do estudo foi a realização das medidas antropométricas por diferentes pesquisadores. Muito embora os avaliadores fossem estudantes de Nutrição e terem sido previamente treinados para realizar as medidas de forma padronizada, valores diferentes entre as avaliações podem comprometer o resultado final do estudo. [14]

Vale ressaltar também que é necessário haver pesquisas com amostras maiores que possam testar associação entre o estado nutricional e o consumo alimentar, visto que, apesar de serem fatores relacionados, não foi possível neste estudo encontrar significância estatística nesta relação. Além disso, outras condições de saúde, como por exemplo a prática de atividade física e outros marcadores, devem ser testados em pesquisas futuras para poder afirmar com mais precisão que o consumo alimentar é um fator determinante.

\section{CONCLUSÃO}

Muito embora a alimentação dos feirantes do município de Dourados tenha tido um aporte de frutas, hortaliças e feijão acima do encontrado em inquéritos brasileiros, como era esperado, o estado nutricional seguiu o padrão nacional, com elevados percentuais de excesso de peso. Ainda que o consumo de marcadores de alimentação saudável tenha sido alto, os alimentos ultraprocessados também estão fortemente presentes na dieta nesta população, sendo influenciado por idade e sexo.

Por fim, os feirantes de Dourados-MS apresentam um perfil socioeconômico e demográfico similar a outros estudos, em que mulheres estão presentes neste setor, pessoas mais velhas e mais escolarizadas, com uma renda média per capita padrão do brasileiro e com a baixa participação de indígenas comercializando seus produtos.

\section{REFERÊNCIAS}

[1] Fante C, Gallina LS, Confortin FG, Lutinski JA. Perfil e preferências dos consumidores de produtos em feiras livres na cidade de Chapecó (SC). Revista Saúde (Sta. Maria). 2020; 46(1).

[2] Brasil. Ministério da Saúde. Guia alimentar para a população brasileira / Ministério da Saúde, Secretaria de Atenção à Saúde, Departamento de Atenção Básica. Brasília: Ministério da Saúde; 2014 (2. Ed).

[3] Rocha HC, Costa C, Castoldi FL, Cecchetti D, Calvete EO, Lodi BS. Perfil socioeconômico dos feirantes e consumidores da Feira do Produtor de Passo Fundo, RS. Ciência Rural. 2010; 40(12): 2593-2597.

[4] Luz VG, Siqueira CEG, La-Rotta EIG, Miquilin IOC, Correa Filho HR. (In)Segurança alimentar e nutricional, autopercepção da saúde e uso de Agrotóxicos: o caso dos agricultores familiares de Ibiúna, São Paulo. Rev Segurança alimentar e nutricional, Campinas. 2015;22(2): 729-741

[5] Instituto Brasileiro de Geografia e Estatística. Pesquisa de orçamentos familiares 2008-2009. Rio de Janeiro: IBGE; 2010.

[6] Instituto Brasileiro de Geografia e Estatística. Pesquisa de orçamentos familiares 2017-2018. Rio de Janeiro: IBGE; 2020.

[7] Brasil. Ministério da Saúde. Vigitel Brasil 2018: vigilância de fatores de risco e proteção para doenças crônicas por inquérito telefônico: estimativas sobre frequência e distribuição sociodemográfica de fatores de risco e proteção para doenças crônicas nas capitais dos 26 estados brasileiros e no Distrito Federal em 2018 / Ministério da Saúde, Secretaria de Vigilância em Saúde, Departamento de Análise em Saúde e Vigilância de Doenças não Transmissíveis. - Brasília: Ministério da Saúde, 2019. 
[8] Instituto Brasileiro de Geografia e Estatística. O Brasil em síntese: conheça as cidades e estados do Brasil. [internet]. 2020 [acesso em 07 jul. 2020]. Disponível em: https://cidades.ibge.gov.br/brasil/ms/dourados/panora $\underline{\mathrm{ma}}$

[9] Instituto de Geografia e Estatística. Pesquisa nacional de saúde do escolar 2012. Rio de Janeiro: IBGE; 2013.

[10] BRASIL. Ministério da Saúde. Secretaria de Atenção à Saúde. Departamento de Atenção Básica. Orientação para a coleta e análise de dados antropométricos em serviços de saúde: Norma Técnica do Sistema de Vigilância alimentar e Nutricional - SISVAN / Ministério da Saúde. Secretaria de Atenção à Saúde. Departamento de Atenção Básica. Brasília: Ministério da Saúde, 2011. 76 p.: il, (Série G. Estatística em Informação a Saúde).

[11] World Health Organization. Obesity: preventing and managing the global epidemic. Report of a WHO Consulation. Geneva: World Health Organization;1998 (Technical Report Series, No. 894).

[12] World Health Organization. Obesity: preventing and managing the global epidemic. Report of a World Health Organization Consultation. Geneva: World Health Organization, 2000. p. 256. WHO Obesity Technical Report Series, n. 284

[13] Blackburn GL, Thornton, PA. Nutritional assessment of the hospitalized patients. Medical Clinics of North America. 1979; (63): 1103-115.

[14] Guedes DP, Guedes JERP. Controle do peso corporal: composição corporal, atividade física e nutrição. 2. ed. Rio de janeiro: Shape, 2003.

[15] Mascarenhas MS. Aspectos psicossociais do trabalho e transtorno mentais comuns entre trabalhadores informais feirantes. (Dissertação de Mestrado). Feira de Santana/BA: Universidade Estadual de Feira de Santana; 2014.

[16] Amor ALM, Silva RM, Silva AAMR, Araújo WC, Oliveira AJ, Almeida JS, et al. Perfil de manipuladores e consumidores de hortaliças provenientes de feiras livres e supermercados. Rev. Baiana de Saúde Pública. 2012 julset; 36(3): 792-815.

[17] Toniasso HR, Souza CC, Brum E, Figueiredo RS. Agricultura familiar e associativismo rural: o caso associação Harmonia de agricultura familiar de mato grosso do sul e a sua sustentabilidade. Informe Gepec. 2007 ju-dez; 12(2): 2-10.

[18] Instituto de Geografia e Estatística. Censo demográfico 2010. Rio de Janeiro: IBGE; 2011.
[19] Instituto de Geografia e Estatística. Pesquisa nacional por amostra de domicílio contínua 2016-2018. Rio de Janeiro: IBGE; 2019.

[20] Brasil. Conselho Nacional de Segurança Alimentar e Nutricional. Tekoha: direitos dos Povos Guarani e Kaiowá: visita do Consea ao Mato Grosso do Sul. Brasília: Presidência da República, 2017.

[21] Mota JGB, Cavalcante TLV. Reserva Indígena de Dourados: Histórias e Desafios Contemporâneos. Ebook, São Leopoldo: Karywa, 2019, 285p.

[22] Instituto de Geografia e Estatística. Pesquisa nacional de saúde 2013. Rio de Janeiro: IBGE; 2015.

[23] Gomes DCK, Sichieri R, Junior EV, Boccolini CS, de Moura Souza A, Cunha DB. Trends in obesity prevalence among Brazilian adults from 2002 to 2013 by educational level. BMC Public Health. 2019; 19(1): 965.

[24] Brasil. Ministério da Saúde. Vigitel Brasil 2016: vigilância de fatores de risco e proteção para doenças crônicas por inquérito telefônico: estimativas sobre frequência e distribuição sociodemográficas de fatores de risco e proteção para doenças crônicas nas capitais dos 26 estados brasileiros e no Distrito Federal em 2016 / Ministério da Saúde, Secretaria de Vigilância em Saúde, Departamento de Vigilância de Doenças e Agravos não Transmissíveis e Promoção da Saúde. Brasília: Ministério da saúde; 2017

[25] Avila JC, Luz VG, Assumpção D, Fisberg RM, Barros MBA. Meat intake among adults: a population-based study in the city of Campinas, Brazil. A cross-sectional study. São Paulo Med. J. [online]. 2016, vol.134, n.2, pp.138-145. Epub Mar 18, 2016. ISSN 18069460. $\underline{3180.2015 .01691609 .}$.

[26] Srour B, Fezeu LK, Kesse-Guyot E, Allès B; Méjean C, Andrianasolo RM. Ultra-processed food intake and risk of cardiovascular disease: prospective cohort study (NutriNet-Santé). BMJ. British Medical Journal (Clinical Research Ed.) 2019; (365): 11451.

[27] Oliveira KSC. Segurança alimentar e nutricional dos agricultores familiares da associação dos produtores e produtoras orgânicas de Ceará MIRIM/RN. (Dissertação de Mestrado). Natal - RN: Universidade Federal do Rio Grande do Norte; 2014.

[28] Alves HJ, Boog MCF. Representações sobre o consumo de frutas, verduras e legumes entre fruticultores de zona rural. Rev. Nutr., Campinas. 2008 nov-dez; 21(6):705-715 